\title{
Author's Correction
}

\section{Development and Evaluation of Multiplex Real-time RT-PCR Assays for Seasonal, Pandemic A/H1pdm09 and Avian A/H5 Influenza Viruses Detection}

\author{
Jang-Hoon Choi, Mi-Seon Kim, Joo-Yeon Lee, Nam-Joo Lee, Donghyok Kwon, \\ Min Gu Kang, and Chun Kang
}

\begin{abstract}
Division of Influenza Virus, Center for Infectious Disease, Korea National Institute of Health,
\end{abstract} Korea Center for Disease Control and Prevention, Osong 363-951, Republic of Korea

In the article by Choi et al. published in Journal of Microbiology 2013; 51, 252-257. Table 1 on page 253 should be changed as below.

Table 1. Primer and probe sets of multiplex real-time RT-PCR assays

\begin{tabular}{|c|c|c|c|c|}
\hline Multiplex set & Target gene & Primer/probe & Sequence $\left(5^{\prime}-3^{\prime}\right)$ & Product size (bp) \\
\hline \multirow{6}{*}{1} & \multirow{3}{*}{$\begin{array}{l}\text { Influenza type A } \\
\text { Matrix }\end{array}$} & Forward & AAT CCT GTC ACC TCT GAC TAA GG & \multirow{3}{*}{99} \\
\hline & & Reverse & CAT TYT GGA CAA AKC GTC TAC G & \\
\hline & & Probe & FAM-TGC AGT CCT CGC TCA C-MGBNFQ & \\
\hline & \multirow{3}{*}{$\begin{array}{l}\text { Influenza type B } \\
\text { Nucleoprotein }\end{array}$} & Forward & GAA TGC TGT CAA TGA ATA TTG AGG G & \multirow{3}{*}{77} \\
\hline & & Reverse & CAT TGA GTC ATT CAT CAT CTT GAG TAG AT & \\
\hline & & Probe & VIC-TCC TTT GAC ATC TGC AT-MGBNFQ & \\
\hline \multirow{9}{*}{2} & \multirow{3}{*}{$\begin{array}{l}\text { A/H1pdm09 } \\
\text { Hemagglutinin }\end{array}$} & Forward & CCC CAA GAC AAG TTC ATG GC & \multirow{3}{*}{88} \\
\hline & & Reverse & TTT GTA GAA GCT TTT TGC TCC AGC & \\
\hline & & Probe & FAM-CAT GAC TCG AAC AAA GG-MGBNFQ & \\
\hline & \multirow{3}{*}{$\begin{array}{l}\text { Avian A/H5 } \\
\text { Hemagglutinin }\end{array}$} & Forward & GGT AAC GGT TGT TTC GAG TTC TAT CA & \multirow{3}{*}{89} \\
\hline & & Reverse & AAT ACT GCG GGT AGT CAT ACG TTC C & \\
\hline & & Probe & NED-ATG TGA TAA TGA ATG TAT GGA AAG T-MGBNFQ & \\
\hline & \multirow{3}{*}{$\mathrm{GAPDH}^{\mathrm{a}}$} & Forward & CCT CCC GCT TCG CTC TCT & \multirow{3}{*}{65} \\
\hline & & Reverse & GCT GGC GAC GCA AAA GA & \\
\hline & & Probe & Cy5-CCT CCT GTT CGA CAG TCA GCC GC- BHQ & \\
\hline \multirow{6}{*}{3} & \multirow{3}{*}{$\begin{array}{l}\text { Seasonal A/H1 } \\
\text { Hemagglutinin }\end{array}$} & Forward & CAA GTG TAA CAG TGA ATG CAT GG & \multirow{3}{*}{150} \\
\hline & & Reverse & GTT GAG TAG ATC GCC AGA ATC TGA TAG & \\
\hline & & Probe & FAM- AGT GAA ATT GGA ATC AAT GG-MGBNFQ & \\
\hline & \multirow{3}{*}{$\begin{array}{l}\text { Seasonal A/H3 } \\
\text { Hemagglutinin }\end{array}$} & Forward & TAG AAA ATG GTT GGG AGG GAA TG & \multirow{3}{*}{102} \\
\hline & & Reverse & CTG CTT GAG TGC TTT TAA GAT CTG C & \\
\hline & & Probe & VIC-TGG TAC GGT TTCA GGC AT-MGBNFQ & \\
\hline
\end{tabular}

